\title{
ORIGINAL ARTICLE \\ Correlation between diffusion tensor imaging parameters and clinical assessments in patients with cervical spondylotic myelopathy with and without high signal intensity
}

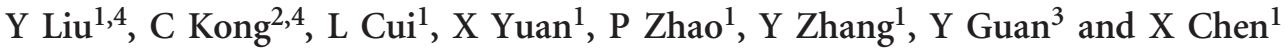

Study design: A cross-sectional observational study.

Objectives: The aim of this study is to compare the apparent diffusion coefficient (ADC) and fractional anisotropy (FA) between patients with cervical spondylotic myelopathy (CSM) with and without high T2-weighted signal intensity, and to correlate each parameter with clinical assessments.

Setting: CSM is a common cause of spinal cord dysfunction. The significance of T2 high signal intensity in the prognosis of CSM remains controversial.

Methods: Diffusion tensor imaging was performed at the cervical spinal cord in 40 patients with CSM and 42 healthy subjects. Patients with high signal intensity were separated from those without high signal intensity. ADC and FA values were compared among different groups, and the correlation between each parameter and the modified Japanese Orthopedic Association (mJOA) score was examined. Results: The ADC and FA values of C2/3 differed significantly from those of $C 5 / 6$ and $C 6 / 7$ in healthy subjects. Patients with CSM had a higher ADC but a lower FA value than did healthy subjects. In all patients with CSM, there was a negative linear correlation between ADC and mJOA score, but FA value correlated positively with mJOA score. Secondary analysis suggested that FA value in patients with high signal intensity was lower than that in patients without high signal intensity. FA value showed a positive linear correlation with mJOA score in the patients with high signal intensity but not in the patients without high signal intensity.

Conclusions: Patients with high signal intensity may have more severe spinal cord injury than patients without high signal intensity, and FA may be a useful indicator of functional status in patients with CSM with high signal intensity.

Spinal Cord (2017) 55, 1079-1083; doi:10.1038/sc.2017.75; published online 5 September 2017

\section{INTRODUCTION}

Cervical spondylotic myelopathy (CSM) is a common disease caused by progressive degenerative vertebral column abnormalities that lead to spinal cord injury. ${ }^{1,2}$ Magnetic resonance imaging (MRI) is the main modality used to diagnose CSM, assess the degree of spinal canal stenosis and evaluate the intramedullary state of the spinal cord. The presence of intramedullary high signal intensity on a T2-weighted image (T2HSI) may reflect chronic spinal cord compression. However, the significance of T2HSI in the prognosis of CSM remains controversial. Some studies suggested that T2HSI indicates a poor prognosis after surgical intervention, ${ }^{3}$ but others showed that T2HSI may not correlate with postoperative neurologic improvement in patients with CSM. ${ }^{4}$

MRI has been widely utilized to assess the degree of spinal cord impairment in patients with chronic traumatic spinal cord injury, multiple sclerosis and spondylotic myelopathy. ${ }^{5-9}$ Although several grading systems have been used to evaluate changes in MRI signal after spinal cord injury, ${ }^{10,11}$ a reliable method to quantitatively assess signal intensity in patients with CSM remains to be established. ${ }^{12-15}$
Diffusion tensor imaging (DTI) is considered to be more advantageous than conventional MRI because it can detect and quantify microstructural abnormalities. ${ }^{5,16-19}$ Recently, Jones et al..$^{20}$ investigated the relationship between DTI parameters and clinical assessment in patients with CSM, and found that fractional anisotropy (FA) value correlated strongly with the modified Japanese Orthopedic Association (mJOA) score and Nurick score. Wen et al. ${ }^{21}$ showed not only that FA correlated positively with mJOA score, but it may also be indicative of surgical outcome with using logistic regression analysis. However, potential functional outcome cannot be inferred by the presence of T2HSI on MRI. In our pilot study, which included only 10 patients with CSM with T2HSI and 10 healthy volunteers, we failed to find any positive correlation, possibly because of the small sample size and the selection bias in patient severity. ${ }^{22}$ Therefore, we expanded the sample size in the current study and incorporated not only patients with T2HSI but also those without T2HSI.

DTI parameters and high signal intensity represent two different modalities for evaluating the neurologic state of the spinal cord. Yet, few researches have compared the two modalities within the same

\footnotetext{
${ }^{1}$ Department of Orthopedics, Beijing Luhe Hospital, Capital Medical University, Beijing, China; ${ }^{2}$ Department of Orthopedics, Beijing Xuanwu Hospital, Capital Medical University, Beijing, China and ${ }^{3}$ Department of Anesthesiology and Critical Care Medicine, Johns Hopkins University, School of Medicine, Baltimore, MD, USA 4 These authors contributed equally to this work and are co-first authors.

Correspondence: Professor Y Guan, Department of Anesthesiology and Critical Care Medicine, Johns Hopkins University, School of Medicine, Baltimore, MD 21205, USA. E-mail: yguan1@jhmi.edu

or Dr X Chen, Department of Orthopedics, Beijing Luhe Hospital, Capital Medical University, 82 Xinhua South Road, Tongzhou District, Beijing 100020, China.

E-mail: xuemingchen@sina.com

Received 11 February 2017; revised 3 May 2017; accepted 22 May 2017; published online 5 September 2017
} 
Table 1 ADC and FA at different levels in healthy subjects

\begin{tabular}{ccc}
\hline Level & $A D C$ & $F A$ \\
\hline $\mathrm{C} 2 / 3$ & $1.211 \pm 0.135$ & $0.678 \pm 0.073$ \\
$\mathrm{C} 3 / 4$ & $1.289 \pm 0.275$ & $0.671 \pm 0.055$ \\
$\mathrm{C} 4 / 5$ & $1.319 \pm 0.343$ & $0.646 \pm 0.085$ \\
$\mathrm{C} 5 / 6$ & $1.395 \pm 0.298$ & $0.590 \pm 0.073$ \\
$\mathrm{C} 6 / 7$ & $1.401 \pm 0.259$ & $0.589 \pm 0.081$ \\
Total & $1.351 \pm 0.251$ & $0.624 \pm 0.046$
\end{tabular}

Abbreviations: ADC, apparent diffusion coefficient; FA, fractional anisotrophy. Each of the data were means \pm s.d. of the 10 healthy subjects. Unit of FA is 1 , unit of ADC is $\times 10^{-3} \mathrm{~mm}^{2} \mathrm{~s}^{-1}$.

study. For the first time, we compared the parameters of ADC and FA between two subgroups of patients with CSM-those with and without high signal intensity - to examine the correlation of each parameter with clinical assessment in these patients. We hypothesized that correlation between certain DTI parameter and mJOA score, which represents an indicator of functional status, differs between patients with and without high signal intensity.

\section{MATERIALS AND METHODS}

\section{Eligibility criteria}

This study was approved by the Ethical Committee of Luhe Hospital, Beijing, China. Forty patients (22 men and 18 women, average age $55.6 \pm 11.9$ years, range 30-69 years) diagnosed with CSM were recruited from February 2011 to February 2015 in Beijing Luhe Hospital (Beijing, China). The average BMI (body mass index) was $26 \pm 3.7 \mathrm{~kg} \mathrm{~m}^{-2}$. All qualifying patients had clinical symptoms (neck pain, stiffness, motion dysfunction, upper extremity weakness or sensory symptoms) that had persisted longer than 6 weeks, and they showed significant spinal cord compression on radiologic examination (ossification of the ligament flavum, cervical disc herniation and cervical spinal stenosis). Patients were excluded if they had previous head or brain lesion associated with trauma, cerebral palsy, rheumatoid arthritis or spine disease including tumors.

Based on signal intensity on cervical T2-weighted MRI images, patients with CSM were further divided into two subgroups: those with high signal intensity (HSI-CSM) and those without high signal intensity (control-CSM). High signal intensity was determined by two experienced surgeons. If the two surgeons had different opinions on evaluation, a third surgeon was called in to help making the final judgment. For the control group, we included 42 age-matched healthy volunteers without any history of neurologic or psychiatric illness (including cervical spine or spinal cord lesion), and without abnormal signal on MRI (no compressive lesions on T2-weighted MRI, including CSM, ossification of the posterior longitudinal ligament, ossification of the ligament flavum, cervical disc herniation and cervical spinal stenosis). The average age of the healthy subjects was 51.2 years (range 24-69). Informed consent was obtained from both patients with CSM and healthy participants.

\section{Image acquisition and processing}

As described in our previous study, ${ }^{22}$ all examinations were performed with a 1.5 T MRI scanner (Signa Excite; GE Medical Systems, Milwaukee, WI, USA). The conventional MRI sequence parameters of the cervical spinal cord were as follows: (1) sagittal T2WI (repetition time $3000 \mathrm{~ms}$, echo time $99 \mathrm{~ms}$, slice/gap $3.0 / 1.0 \mathrm{~mm}$, matrix $320 \times 224$, number of excitation $=2$ ); (2) sagittal T1WI (repetition time $410 \mathrm{~ms}$, echo time $9.9 \mathrm{~ms}$, slice/gap $3.0 / 1.0 \mathrm{~mm}$, matrix $320 \times 192$, number of excitation $=3$ ); and (3) axial T2WI (repetition time $3500 \mathrm{~ms}$, echo time $117 \mathrm{~ms}$, slice/gap 4.0/0.5 mm, matrix $288 \times 192$, number of excitation=3). In DTI scans, we used a single-shot spin-echo echo planar image with a sense factor of 4 for the cervical spinal cord with the following parameters: b-value of $500 \mathrm{~mm}^{2} \mathrm{~s}^{-1}$ and $0 \mathrm{~mm}^{2} \mathrm{~s}^{-1}, 15$ diffusion gradient directions, acquisition matrix $128 \times 128$ and field of view $240 \times 240 \mathrm{~mm}$.

A physician performed the region of interest (ROI) measurements using the commercially available software ADW 4.3 (GE Medical Systems), which has
Table 2 Comparisons of ADC and FA among different groups

\begin{tabular}{lccc}
\hline Healthy subjects & $\begin{array}{c}\text { Patients without } \\
\text { high signal intensity }\end{array}$ & $\begin{array}{c}\text { Patients with } \\
\text { high signal intensity }\end{array}$ \\
\hline ADC & $1.351 \pm 0.251$ & $1.947 \pm 0.463$ & $2.028 \pm 0.508$ \\
FA & $0.624 \pm 0.046$ & $0.484 \pm 0.085$ & $0.422 \pm 0.067$ \\
\hline
\end{tabular}

Abbreviations: ADC, apparent diffusion coefficient; FA, fractional anisotrophy.

Each of the data were mean \pm s.d. Unit of FA is 1 , unit of ADC is $\times 10^{-3} \mathrm{~mm}^{2} \mathrm{~s}^{-1}$

been described in detail in our previous published article. ${ }^{22}$ In the healthy subjects, region of interest was placed at five levels from $\mathrm{C} 2 / \mathrm{C} 3$ to $\mathrm{C} 6 / \mathrm{C} 7$, with the sagittal T2-weighted images used as references. For healthy subjects, five region of interests were chosen at each level with caution to avoid partial volume effects from cerebrospinal fluid. All the measurements were averaged across five region of interests for each level, and measurements for each healthy subject were averaged across five levels. For patients with CSM, five region of interests were drawn at the lesion with the most severe compression on T2-weighted MRI.

\section{Functional assessment}

All patients with CSM were evaluated by the mJOA score, which has been validated as a reliable outcome measurement in patients with CSM. A mJOA score between 15 and 17 was defined as mild symptomatology, a score between 11 and 14 was defined as moderate symptomatology and a score lower than 11 was considered severe.

\section{Statistical analysis}

Statistical analysis was carried out with SPSS version 18.0 (Chicago, IL, USA). One-way analysis of variances was used to compare data among healthy subjects, patients without high signal intensity and patients with high signal intensity. The Tukey honest significant difference post hoc test was used to compare specific data points. Bonferroni correction was applied for multiple comparisons. Correlations of DTI metrics with mJOA scores were calculated with Spearman's correlation analysis. Two-tailed tests were performed, and numerical data are expressed as mean \pm s.e.m.; $P<0.05$ was considered significant in all the tests.

\section{RESULTS}

Cervical spinal cord DTI parameters in healthy subjects

In healthy subjects, $\mathrm{ADC}$ value showed an ascending trend from $\mathrm{C} 2 / 3$ to $\mathrm{C6/7}$ of cervical spinal cord, whereas FA value showed a descending trend. The ADC and FA values of $\mathrm{C} 2 / 3$ were significantly different from those of $\mathrm{C} 6 / 7$ (Table 1, $\mathrm{ADC}$ : $\mathrm{F}=3.546, P=0.008$; $\mathrm{FA}: \mathrm{F}=13.82$, $P<0.001)$. The average age and BMI were not significantly different among healthy subjects, patients without high signal intensity and patients with high signal intensity. $(P \geqslant 0.352)$.

\section{Comparisons of DTI parameters among different groups}

DTI parameters at different cervical levels were averaged in healthy subjects for comparison with those of patients with CSM. The ADC values at the most severe compression levels in patients with high signal intensity and in patients without high signal intensity were significantly greater than those of the healthy subjects (Table 2, $P<0.001$ ). The ADC values were not significantly different between patients with high signal intensity and patients without high signal intensity $(P=0.767)$. The FA values in all patients with CSM were significantly lower than those of healthy subjects $(P<0.001)$. The FA value in the patients with high signal intensity was lower than that in the patients without high signal intensity. 
a

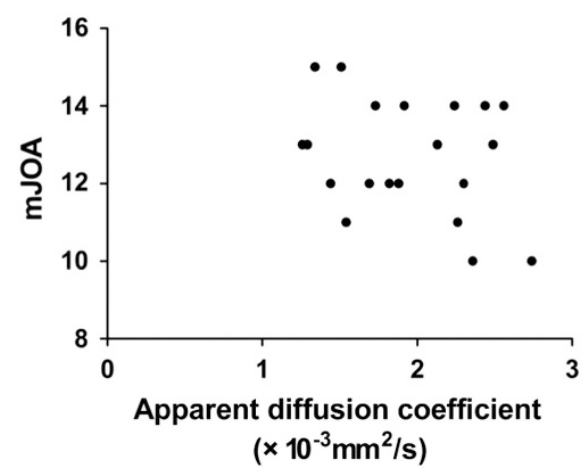

b

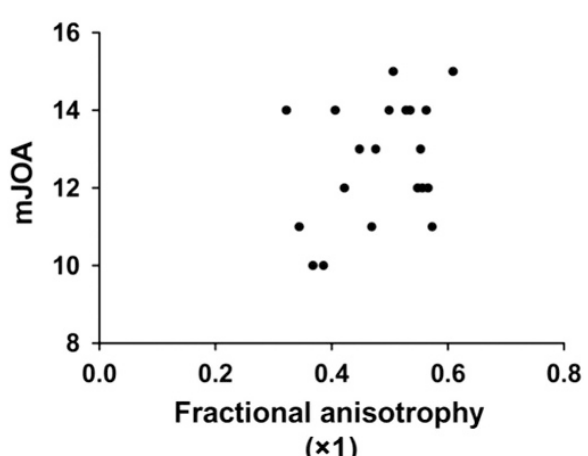

$(\times 1)$

Figure 1 (a) Scatter plot of apparent diffusion coefficient (ADC) values and modified Japanese Orthopedic Association (mJOA) scores in patients without high signal intensity (control-CSM; $n=20$ ). (b) Scatter plot of fractional anisotropy (FA) values and mJOA scores in patients without high signal intensity (control-CSM). There was no correlation of ADC $(r=-0.287, P=0.220)$ or FA $(r=0.329, P=0.156)$ with mJOA score.

a

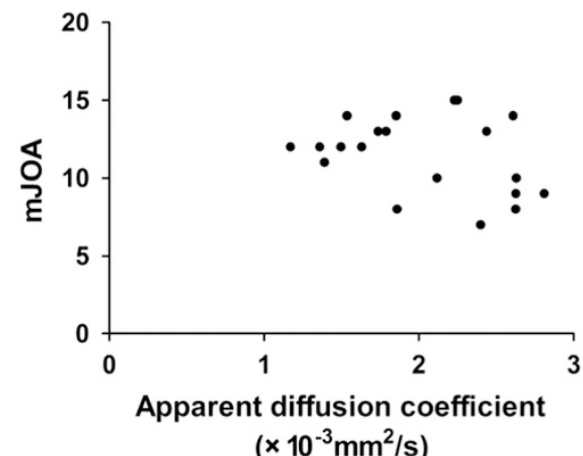

b

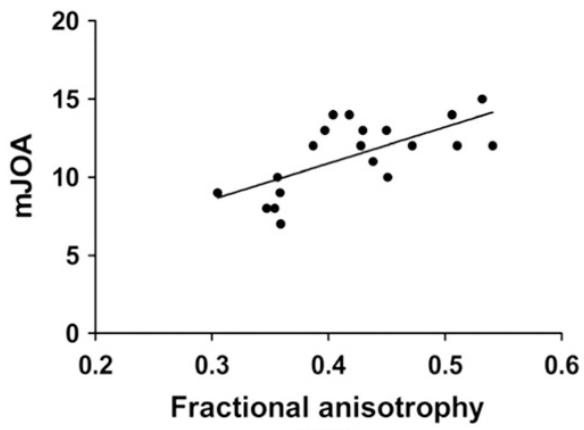

$(\times 1)$

Figure 2 (a) Scatter plot showed no correlation between apparent diffusion coefficient (ADC) values and modified Japanese Orthopedic Association (mJOA) scores ( $r=-0.287, P=0.220, n=20)$ in patients with high signal intensity (HSI-CSM). (b) There is a positive linear correlation between fractional anisotropy (FA) values and mJOA scores $(r=0.359, P=0.005)$ in patients with high signal intensity (HSI-CSM).

a

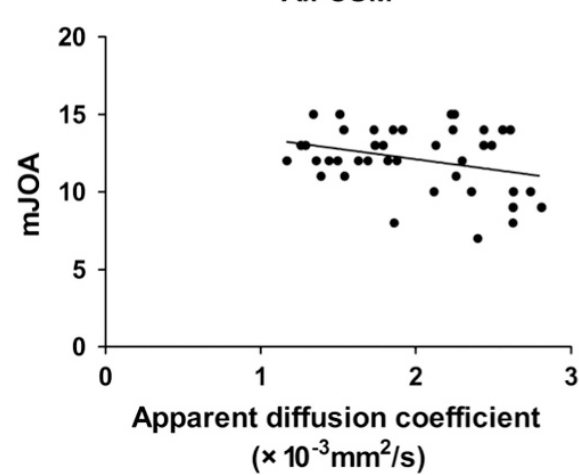

b

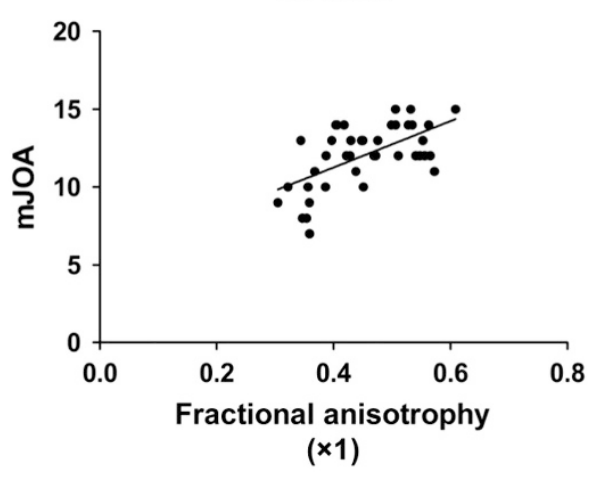

Figure 3 (a) When all patients with CSM were combined, scatter plots showed a negative linear correlation between apparent diffusion coefficient (ADC) values and modified Japanese Orthopedic Association (mJOA) scores $(n=40 ; r=-0.3121, P=0.049)$. (b) However, there was a positive linear coefficient between fractional anisotropy (FA) values and mJOA scores $(r=0.5539, P=0.0002)$

Correlation between DTI indexes and mJOA scores in patients with CSM

The mJOA scores of patients without high signal intensity were significantly higher than those in the patients with high signal intensity $(P=0.034)$. Neither FA $(r=0.329, P=0.156)$ nor ADC $(r=-0.287$,
$P=0.220)$ showed correlation with mJOA scores in patients without high signal intensity (Figure 1). However, in patients with high signal intensity, FA values showed a significant positive correlation with mJOA score $(r=0.359, P=0.005$, Figure 2$)$, whereas ADC values did not $(r=-0.287, P=0.220)$. When we combined all patients' data, 
both FA values $(r=0.5539, P=0.0002)$ and ADC values $(r=-0.3121$, $P=0.049)$ showed significant linear correlations with $\mathrm{mJOA}$ scores (Figure 3).

\section{DISCUSSION}

In 1987, Takahashi et al. ${ }^{23}$ first described T2 high signal intensity of the spinal cord in patients with CSM, and suggested that it might correlate with postoperative prognosis. Because T2 high signal intensity was thought to reflect pathologic changes in the spinal cord, it was extensively studied in patients with CSM for its potential to indicate surgical outcome., ${ }^{3,24}$ However, other investigators have challenged this notion. ${ }^{25,26}$ This disparity may be due in part to previous studies that focused merely on changes in signal intensity and their direct correlation with surgical outcome but did not carefully grade the changes in MRI signal that might reflect injury severity. ${ }^{27}$ Different methods have been used in previous studies to quantify changes in signal intensity and examine their relevance to clinical outcome. For example, T2 high signal intensity was initially quantified by into five grades of ascending severity ( 0 , none; to 4, very intense). Yet, as this grading system was overly meticulous for classifying signal changes, some investigators preferred to use a more simplified scale (0, absent; 1, obscure; and 2, bright). ${ }^{26,28}$ Chen et al. ${ }^{29}$ suggested that Type 2 T2 high signal intensity might indicate a poorer surgical outcome than Type 1. Although in an earlier study, Yukawa et al. ${ }^{30}$ proposed that classification of T2 high signal intensity may be a good predictor of surgical outcome, their recent study failed to demonstrate significant correlation between the two. ${ }^{31}$ Several reasons may account for this apparent discrepancy. For instance, although MRI can help doctors to assess morphologic changes and the intramedullary state of the spinal cord, it is very difficult to use preoperative MRI to predict future functional recovery of the spinal cord without quantitative analysis. Furthermore, because the sequence of parameters is often individually selected for each patient, MRI signal intensity varies greatly between subjects. ${ }^{4}$

DTI is a reliable method for quantifying the microstructural abnormalities of the cervical spinal cord in patients with CSM, ${ }^{5,17-19}$ and many have suggested the correlation of DTI parameters with clinical severity. ${ }^{20,21}$ In our previous study, we confirmed that DTI parameters of the cervical spinal cord in patients with high signal intensity were significantly different from those in healthy subjects. ${ }^{22}$ DTI parameters and T2 high signal intensity are two different methods to evaluate the neurologic state of the spinal cord. So far, no study has compared DTI parameters between patients with CSM with high signal intensity and those without high signal intensity. Our current study showed for the first time that FA value in patients with high signal intensity was significantly lower than that of the patients without high signal intensity. In contrast, ADC values showed no significant difference between the two groups. These novel findings suggest that patients with high signal intensity may have more severe intramedullary injury than those without high signal intensity, and that FA value may be a useful indicator of injury severity. Interestingly, DTI is believed to detect pathologic changes of the white matter, whereas T2 high signal intensity may mostly reflect pathologic changes of the gray matter. ${ }^{32,33} \mathrm{Al}-\mathrm{Mefty}$ et al. $^{32}$ reported that intramedullary changes of the spinal cord on MRI coincided with pathologic changes in the gray matter, which includes loss of motor neurons, necrosis and cavitation. Ohshio et al. ${ }^{33}$ suggested that the signal pattern of $\mathrm{T} 1$ hypointensity/T2 hyperintensity changes indicated necrosis, myelomalacia and spongiform change in the gray matter. It is possible that because white matter is likely to be compressed first in patients with CSM, gray matter injury would then indicate that severe white matter injury has occurred.

Neither ADC nor FA value exhibited a correlation with mJOA scores in patients without high signal intensity. However, FA value showed a strong positive correlation with mJOA score in patients with high signal intensity. In light of potential selection bias of patient severity when performing linear correlation analysis in each subgroup, we also combined all patients' data for analysis. FA value showed a significant positive correlation with mJOA score in all patients with CSM; hence FA may be a useful indicator of functional status in patients with CSM, especially those with high signal intensity. Current finding is in line with the notion that FA may be a sensitive biomarker for axonal dysfunction and demyelination. ${ }^{34}$ Our study may have some limitations, including the limited sample size, suboptimal spatial resolution and signal-to-noise ratio with the $1.5 \mathrm{~T}$ MRI scanner. A future study with a large sample size that includes postoperative neurologic assessment of patients with CSM is warranted. In addition, as high signal intensity is a qualitative parameter rather than a quantitative parameter, inter/intra-observer reliability analysis of the data will also be helpful to correlate the analysis between DTI parameters and postoperative clinical score.

\section{DATA ARCHIVING}

There were no data to deposit.

\section{CONFLICT OF INTEREST}

The authors declare no conflict of interest.

\section{ACKNOWLEDGEMENTS}

We thank Claire F Levine, MS, ELS (scientific editor, Department of Anesthesiology/CCM, Johns Hopkins University) for editing the manuscript. This study was supported by grant from the Science and Technology Commission of Tongzhou District. This study was funded by Beijing Natural Science Foundation Program and Scientific Research Key Program of Beijing Municipal Commission of Education (KM 201710025028) and Capital Characteristic Clinical Project (Z161100000116064).

1 Irwin ZN, Hilibrand A, Gustavel M, McLain R, Shaffer W, Myers M et al. Variation in surgical decision making for degenerative spinal disorders. Part II: cervical spine. Spine 2005; 30: 2214-2219.

2 Holly LT, Matz PG, Anderson PA, Groff MW, Heary RF, Kaiser MG et al. Clinical prognostic indicators of surgical outcome in cervical spondylotic myelopathy. J Neurosurg Spine 2009; 11: 112-118.

3 Zhang P, Shen Y, Zhang YZ, Ding WY, Wang LF. Significance of increased signal intensity on MRI in prognosis after surgical intervention for cervical spondylotic myelopathy. J Clin Neurosci 2011; 18: 1080-1083.

4 Uchida K, Nakajima H, Takeura N, Yayama T, Guerrero AR, Yoshida A et al. Prognostic value of changes in spinal cord signal intensity on magnetic resonance imaging in patients with cervical compressive myelopathy. Spine J 2014; 14: 1601-1610.

5 Ellingson BM, Salamon N, Holly LT. Advances in MR imaging for cervical spondylotic myelopathy. Eur Spine J 2013; 45: 432-438.

6 Kelley BJ, Harel NY, Kim CY, Papademetris X, Coman D, Wang X et al. Diffusion tensor imaging as a predictor of locomotor function after experimental spinal cord injury and recovery. J Neurotrauma 2014; 31: 1362-1373.

7 Jones JG, Cen SY, Lebel RM, Hsieh PC, Law M. Diffusion tensor imaging correlates with the clinical assessment of disease severity in cervical spondylotic myelopathy and predicts outcome following surgery. AJNR Am J Neuroradiol 2013; 34: 471-478.

8 Petersen JA, Wilm BJ, von Meyenburg J, Schubert M, Seifert B, Najafi Y et al. Chronic cervical spinal cord injury: DTI correlates with clinical and electrophysiological measures. J Neurotrauma 2012; 29: 1556-1566.

9 Oh J, Zackowski K, Chen M, Newsome S, Saidha S, Smith SA et al. Multiparametric MRI correlates of sensorimotor function in the spinal cord in multiple sclerosis. Mult Scler 2013; 19: 427-435. 
10 Li F, Chen Z, Zhang F, Shen H, Hou T. A meta-analysis showing that high signal intensity on T2-weighted MRI is associated with poor prognosis for patients with cervical spondylotic myelopathy. J Clin Neurosci 2011; 18: 1592-1595.

11 Yukawa Y, Kato F, Yoshihara H, Yanase M, Ito K. MR T2 image classification in cervical compression myelopathy: predictor of surgical outcomes. Spine (Phila Pa 1976) 2007; 32: $1675-1678$.

12 Alafifi T, Kern R, Fehlings M. Clinical and MRI predictors of outcome after surgical intervention for cervical spondylotic myelopathy. J Neuroimaging 2007; 17: 315-322.

13 Mastronardi L, Elsawaf A, Roperto R, Bozzao A, Caroli M, Ferrante M et al. Prognostic relevance of the postoperative evolution of intramedullary spinal cord changes in signal intensity on magnetic resonance imaging after anterior decompression for cervical spondylotic myelopathy. J Neurosurg Spine 2007; 7: 615-622.

14 Matsumoto M, Toyama Y, Ishikawa M, Chiba K, Suzuki N, Fujimura Y. Increased signal intensity of the spinal cord on magnetic resonance images in cervical compressive myelopathy. Does it predict the outcome of conservative treatment? Spine (Phila Pa 1976) 2000; 25: 677-682.

15 Suri A, Chabbra RP, Mehta VS, Gaikwad S, Pandey RM. Effect of intramedullary signal changes on the surgical outcome of patients with cervical spondylotic myelopathy. Spine J 2003; 3: 33-45.

16 Budzik JF, Balbi V, Le Thuc V, Duhamel A, Assaker R, Cotten A. Diffusion tensor imaging and fibre tracking in cervical spondylotic myelopathy. Eur Radiol 2011; 21: 426-433.

17 Ellingson BM, Ulmer JL, Kurpad SN, Schmit BD. Diffusion tensor MR imaging of the neurologically intact human spinal cord. AJNR Am J Neuroradiol 2008; 29: 1279-1284.

18 Zhang C, Das SK, Yang DJ, Yang HF. Application of magnetic resonance imaging in cervical spondylotic myelopathy. World J Radiol 2014; 6: 826-832.

19 Staempfli P, Reischauer C, Jaermann T, Valavanis A, Kollias S, Boesiger P. Combining fMRI and DTI: a framework for exploring the limits of fMRI-guided DTI fiber tracking and for verifying DTI-based fiber tractography results. Neuroimage 2008 ; 39: 119-126.

20 Jones J, Lerner A, Kim PE, Law M, Hsieh PC. Diffusion tensor imaging in the assessment of ossification of the posterior longitudinal ligament: a report on preliminary results in 3 cases and review of the literature. Neurosurg Focus 2011; 30: E14.

21 Wen CY, Cui JL, Liu HS, Mak KC, Cheung WY, Luk KD et al. Is diffusion anisotropy a biomarker for disease severity and surgical prognosis of cervical spondylotic myelopathy. Radiology 2014; 270: 197-204.
22 Chen X, Kong C, Feng S, Guan H, Yu Z, Cui L et al. Magnetic resonance diffusion tensor imaging of cervical spinal cord and lumbosacral enlargement in patients with cervical spondylotic myelopathy. J Magn Reson Imaging 2016; 43: 1484-1491.

23 Takahashi M, Sakamoto Y, Miyawaki M, Bussaka H. Increased MR signa intensity secondary to chronic cervical cord compression. Neuroradiology 1987; 29: 550-556.

24 Al-Mefty O, Harkey LH, Middleton TH, Smith RR, Fox JL. Myelopathic cervical spondylotic lesions demonstrated by magnetic resonance imaging. J Neurosurg 1988; 68: 217-222.

25 Naderi S, Ozgen S, Pamir MN, Ozek MM, Erzen C. Cervical spondylotic myelopathy: surgical results and factors affecting prognosis. Neurosurgery 1998; 43: 43-50.

26 Nakamura M, Fujimura Y. Magnetic resonance imaging of the spinal cord in cervical ossification of the posterior longitudinal ligament: can it predict surgical outcome? Spine 1998; 23: 38-40.

27 Avadhani A, Rajasekaran S, Shetty AP. Comparison of prognostic value of different MRI classifications of signal intensity change in cervical spondylotic myelopathy. Spine J 2010; 10: 475-485.

28 Hirabayashi K, Miyakawa J, Satomi K, Maruyama T, Wakano K. Operative results and postoperative progression of ossification among patients with ossification of cervical posterior longitudinal ligament. Spine 1981; 6: 354-364.

29 Chen CJ, Lyu RK, Lee ST, Wong YC, Wang LJ. Intramedullary high signal intensity on T2-weighted MR images in cervical spondylotic myelopathy: prediction of prognosis with type of intensity. Radiology 2001; 221: 789-794.

30 Yukawa Y, Kato F, Yoshihara H, Yanase M, Ito K. MR T2 image classification in cervical compression myelopathy: predictor of surgical outcomes. Spine 2007; 32: 1675-1679.

31 Yukawa $\mathrm{Y}$, Kato $\mathrm{F}$, Ito $\mathrm{K}$, Horie $\mathrm{Y}$, Hida $\mathrm{T}$, Machino $\mathrm{M}$ et al. Postoperative changes in spinal cord signal intensity in patients with cervical compression myelopathy: comparison between preoperative and postoperative magnetic resonance images. J Neurosurg Spine 2008; 8: 524-528.

32 Al-Mefty O, Harkey HL, Marawi I, Haines DE, Peeler DF, Wilner HI et al. Experimental chronic compressive cervical myelopathy. J Neurosurg 1993; 79: 550-561.

33 Ohshio I, Hatayama A, Kaneda K, Takahara M, Nagashima K. Correlation between histopathologic features and magnetic resonance images of spinal cord lesions. Spine 1993; 18: 1140-1149.

34 Vedantam A, Eckardt G, Wang MC, Schmit BD, Kurpad SN. Clinical correlates of high cervical fractional anisotropy in acute cervical spinal cord injury. World Neurosurg 2015; 83: 824-828. 